Research Article

\title{
Novel Stability Results for Caputo Fractional Differential Equations
}

\author{
Abdellatif Ben Makhlouf $(\mathbb{D}$ and El-Sayed El-Hady \\ Mathematics Department, College of Science, Jouf University, P.O. Box 2014, Sakaka, Saudi Arabia \\ Correspondence should be addressed to Abdellatif Ben Makhlouf; abmakhlouf@ju.edu.sa
}

Received 8 May 2021; Accepted 19 June 2021; Published 1 July 2021

Academic Editor: Amr Elsonbaty

Copyright ( $\odot 2021$ Abdellatif Ben Makhlouf and El-Sayed El-Hady. This is an open access article distributed under the Creative Commons Attribution License, which permits unrestricted use, distribution, and reproduction in any medium, provided the original work is properly cited.

\begin{abstract}
Modelling some diseases with large mortality rates worldwide, such as COVID-19 and cancer is crucial. Fractional differential equations are being extensively used in such modelling stages. However, exact analytical solutions for the solutions of such kind of equations are not reachable. Therefore, close exact solutions are of interests in many scientific investigations. The theory of stability in the sense of Ulam and Ulam-Hyers-Rassias provides such close exact solutions. So, this study presents stability results of some Caputo fractional differential equations in the sense of Ulam-Hyers, Ulam-Hyers-Rassias, and generalized Ulam-Hyers-Rassias. Two examples are introduced at the end to show the validity of our results. In this way, we generalize several recent interesting results.
\end{abstract}

\section{Introduction and Preliminaries}

Fractional calculus provides a powerful tool in both theoretical frameworks and practical aspects. In many disciplines, fractional modelling is much more suitable than the classical one. This is because of the nice modelling tools that are available only in fractional calculus (see e.g., [1,2]). In particular, fractional calculus has been used extensively in the modelling stages in the fields of economics, chemistry, aerodynamics, physics, and polymer rheology. It should be remarked also that a certain kind of fractional derivative has been used recently to model Ebola virus (see [3]) and HIV (see [4]). Fractional differential equations with Caputo and Caputo-Fabrizio derivatives are used recently by the authors in [5] for the model of cancer-immune system.

The stability issue has gained substantially important attention in several research fields through applications. There are many kinds of stability; one of them is the stability introduced by Ulam in 1940. Since then, the problem is known as Ulam-Hyers stability or simply Ulam stability (see e.g., [6], for more details). Its applications for many types of equations have been investigated by many researchers. For more details on this concept, the readers can see the interesting works [7-10]. The stability problem that is introduced by Ulam can be stated as follows.

Assume that $\mathbb{G}_{1}$ is a group and $\left(\mathbb{G}_{2}, \chi\right)$ a metric group. Given some $\varepsilon^{*}>0$, does there exist $\delta^{*}>0$ such that if $F: \mathbb{G}_{1} \longrightarrow \mathbb{G}_{2}$ satisfies

$$
\chi\left(F\left(x_{1} x_{2}\right), F\left(x_{1}\right) F\left(x_{2}\right)\right)<\delta^{*},
$$

for all $x_{1}, x_{2} \in \mathbb{G}_{1}$, then a homomorphism $F^{*}: \mathbb{G}_{1} \longrightarrow \mathbb{G}_{2}$ exists such that

$$
\chi\left(F\left(x_{1}\right), F^{*}\left(x_{1}\right)\right)<\varepsilon^{*},
$$

for all $x_{1} \in \mathbb{G}_{1}$ ?

Ulam's problem has been extended in many directions for interesting settings. In particular, Rassias (see [11]) generalized Ulam's result for Banach spaces. The nice result of Rassias reads as follows (see [11]).

Theorem 1. Consider Banach spaces $\mathbb{B}$ and $\mathbb{B}^{*}$, and suppose a mapping $Y: \mathbb{B} \longrightarrow \mathbb{B}^{*}$ such that the function $t \mapsto \Upsilon(t x)$ from $\mathbb{R}$ into $\mathbb{B}^{*}$ is continuous for each fixed $x \in \mathbb{B}$. Assume that there are some $\beta \geq 0$ and $\omega \in[0,1)$, fulfilling 
$\left\|\Upsilon\left(x_{1}+x_{2}\right)-\Upsilon\left(x_{1}\right)-\Upsilon\left(x_{2}\right)\right\| \leq \beta\left(\left\|x_{1}\right\|^{\omega}+\left\|x_{2}\right\|^{\omega}\right), \quad x_{1}, x_{2} \in \mathbb{B} \backslash\{0\}$.

Then, a unique solution exists $\Upsilon^{*}: \mathbb{B} \longrightarrow \mathbb{B}^{*}$ of the Cauchy equation $\left(F\left(x_{1}+x_{2}\right)=F\left(x_{1}\right)+F\left(x_{2}\right)\right)$ with

$$
\left\|\Upsilon\left(x_{1}\right)-\Upsilon^{*}\left(x_{1}\right)\right\| \leq \frac{2 \beta\left\|x_{1}\right\|^{\omega}}{\left|2-2^{\omega}\right|}, \quad x_{1} \in \mathbb{B} \backslash\{0\} .
$$

The theorem of Rassias (see [11]) is nowadays known as the Hyers-Ulam-Rassias stability.

Throughout the study, we denote the set of reals by $\mathbb{R}$, the set of nonzero reals by $\mathbb{R}^{*}$, and the set of complex numbers by $\mathbb{C}$, and we fix an interval $I:=[\nu, \nu+T]$ for some reals $\nu, T$ with $T>0$.

Definition 1. Let $\tilde{\lambda}>0, \chi \in \mathbb{C}$. The Mittag-Leffler function (MLF) (see e.g., [2]) $\mathbb{E}_{\tilde{\lambda}}$ is defined as

$$
\mathbb{E}_{\tilde{\lambda}}(\chi):=\sum_{n=0}^{\infty} \frac{\chi^{n}}{\Gamma(\widetilde{\lambda} n+1)} .
$$

Similar to the exponential function, the function $h(s)=$ $\mathbb{E}_{\tilde{\lambda}}\left(M(s-c)^{\tilde{\lambda}}\right)$ satisfies ${ }^{C} D_{c, s}^{\tilde{\lambda}} h(s)=M h(s)$ and $I_{c}^{\tilde{\lambda}} h(s)=$ $(1 / M)(h(s)-1)$, where $M \in \mathbb{R}^{*}$.

Remark 1. Authors in [12-15] obtained some stability results by using the MLF.

Now, we present the notion of generalized metric as follows. Let $Z$ be a nonempty set.

Definition 2. A mapping $9: Z \times Z \longrightarrow[0, \infty]$ is called a generalized metric on $Z$ if and only if $\vartheta$ satisfies the following:

$$
\begin{aligned}
& \text { G1 } \vartheta\left(\xi_{1}, \xi_{2}\right)=0 \text { if and only if } \xi_{1}=\xi_{2} \\
& \text { G2 } \vartheta\left(\xi_{1}, \xi_{2}\right)=\vartheta\left(\xi_{2}, \xi_{1}\right) \text { for all } \xi_{1}, \xi_{2} \in Z \\
& \text { G3 } \vartheta\left(\xi_{1}, \xi_{3}\right) \leq \vartheta\left(\xi_{1}, \xi_{2}\right)+\vartheta\left(\xi_{2}, \xi_{3}\right) \text { for all } \xi_{1}, \xi_{2}, \xi_{3} \in Z
\end{aligned}
$$

The notion of stability in the sense of Ulam-Hyers (UH), Ulam-Hyers-Rassias (UHR), and generalized UHR of fractional differential equations can be introduced as follows (see e.g., [16]). We consider the following fractional differential equation:

$$
H\left(t, \kappa,{ }^{C} D_{\nu, t}^{\tilde{\lambda}} \kappa(t)\right)=0,
$$

and the following three inequalities:

$$
\begin{aligned}
& \left|H\left(t, \kappa,{ }^{C} D_{\nu, t}^{\tilde{\lambda}} \kappa(t)\right)\right| \leq \varepsilon, \\
& \left|H\left(t, \kappa,{ }^{C} D_{\nu, t}^{\tilde{\lambda}} \kappa(t)\right)\right| \leq \varrho(t), \\
& \left|H\left(t, \kappa,{ }^{C} D_{\nu, t}^{\tilde{\lambda}} \kappa(t)\right)\right| \leq \varepsilon \varrho(t),
\end{aligned}
$$

We define the stability of (6) as follows.
Definition 3. Equation (6) is called stable in the sense of $\mathrm{UH}$ if for a given $\varepsilon>0$ and a function $\kappa$ which satisfies (7), and there exists a solution $\kappa_{0}$ of (6) such that

$$
\left|\kappa\left(t_{1}\right)-\kappa_{0}\left(t_{1}\right)\right| \leq c \varepsilon .
$$

Definition 4. Equation (6) is called UHR stable if, for some $\varepsilon>0$ and a function $\kappa$ satisfying (9), there exists a solution $\kappa_{0}$ of (6) such that

$$
\left|\kappa\left(t_{1}\right)-\kappa_{0}\left(t_{1}\right)\right| \leq c \varepsilon \varrho\left(t_{1}\right),
$$

where $\varrho\left(t_{1}\right)$ is some positive, nondecreasing, and continuous function.

Definition 5. Equation (6) is called generalized UHR stable if, for some $\varepsilon>0$ and a function $\kappa$ satisfying (8), there exists a solution $\kappa_{0}$ of (6) such that

$$
\left|\kappa\left(t_{1}\right)-\kappa_{0}\left(t_{1}\right)\right| \leq c \varrho\left(t_{1}\right)
$$

The theorem below represents a basic well-known fixed point theory (see [17]). This theorem plays a fundamental role in our study.

Theorem 2. Assume that $(P, R)$ is a metric space that is generalized completely. Let $M: P \longrightarrow P$ be a strictly contractive operator. If there is an integer $u \geq 0$ with $R\left(M^{u+1} d, M^{u} d\right)<\infty$ for some $d \in P$, therefore,

(a) $\lim _{l \longrightarrow+\infty} M^{l} d=d^{*}$, where $d^{*}$ is the unique fixed point of $M$ in

$$
P^{*}:=\left\{d_{1} \in P: R\left(M^{u} d, d_{1}\right)<\infty\right\} .
$$

(b) If $d_{1} \in P^{*}$, then $R\left(d_{1}, d^{*}\right) \leq(1 /(1-\widetilde{K})) R\left(M d_{1}, d_{1}\right)$.

Define the space $X$ as $X:=C(I, \mathbb{R})$.

Lemma 1. Define a metric $d: X \times X \longrightarrow[0, \infty]$ in such $a$ way that

$$
d\left(\kappa_{1}, \kappa_{2}\right)=\inf \left\{\widetilde{D} \in[0, \infty]: \frac{\left|\kappa_{1}(s)-\kappa_{2}(s)\right|}{\mathbb{E}_{\tilde{\lambda}}\left(\tilde{\eta}(s-\nu)^{\tilde{\lambda}}\right)} \leq \widetilde{D} \beta(s), s \in I\right\},
$$

where $\tilde{\eta}>0$ and $\beta \in C(I,(0,+\infty))$. Then, $(X, d)$ is a generalized complete metric space.

Remark 2. Note that the authors in [18] proved the existence and uniqueness of global solutions using the norm:

$$
\|\kappa\|_{\gamma}=\sup _{l \in[0, \vartheta]} \frac{\|\kappa(l)\|}{\mathbb{E}_{\tilde{\lambda}}\left(\gamma l^{\lambda}\right)}, \quad \text { for } \kappa \in C\left([0, \vartheta], \mathbb{R}^{d}\right) \text {. }
$$

This contribution is considered as a generalized version of the interesting results in [19-21]. Our contribution is original for many reasons. First, the metric used is a function of the Mittag-Leffler function. Second, the obtained results are in a complete generalized metric space. Third, the tool 
used is a version of Banach fixed point theory. The main purpose of this study is to study the stability of the following initial value problem:

$$
\begin{aligned}
{ }^{C} D_{\nu, \omega}^{\tilde{\lambda}} y(\omega) & =G(\omega, y(\omega)), \\
y(\nu) & =y_{v},
\end{aligned}
$$

in the sense depicted in Definitions 3-5, where $\widetilde{\lambda} \in(0,1)$, ${ }^{C} D_{\nu, \omega}^{\tilde{\lambda}}$ is the Caputo fractional derivative, and $G: I \times \mathbb{R} \longrightarrow \mathbb{R}$ is a given function. It should be noted that the solution of the initial value problem (16) is given by

$$
y(\omega)=y_{\nu}+\frac{1}{\Gamma(\widetilde{\lambda})} \int_{\nu}^{\omega}(\omega-\varsigma)^{\tilde{\lambda}-1} G(\varsigma, y(\varsigma)) \mathrm{d} \varsigma .
$$

\section{Stability Results}

In this section, we present our main results. In other words, we prove that, under certain conditions, functions that satisfy (16) approximately (in some sense) are close (in some way) to the solutions of (16). We have done this in both $\mathrm{UH}$ sense and also in UHR sense. The following theorem represents the stability of (16) in the sense of UHR.

Theorem 3. Assume $G: I \times \mathbb{R} \longrightarrow \mathbb{R}$ is continuous and satisfies

$$
\left|G\left(\omega, \kappa_{1}\right)-G\left(\omega, \kappa_{2}\right)\right| \leq L_{G}\left|\kappa_{1}-\kappa_{2}\right|,
$$

for all $\omega \in I, \kappa_{i} \in \mathbb{R}, i=1,2$, and for some $L_{G}>0$. If an absolutely continuous function $x: I \longrightarrow \mathbb{R}$ satisfies

$$
\left|{ }^{C} D_{\gamma, \omega}^{\tilde{\lambda}} x(\omega)-G(\omega, x(\omega))\right| \leq \varepsilon(\omega),
$$

for all $\omega \in I$, where $\varepsilon>0$ and $\varrho(\omega)$ is a positive, nondecreasing, and continuous function, then there is a solution $x^{*}$ of (16) such that

$$
\left|x(\omega)-x^{*}(\omega)\right| \leq\left(\frac{L_{G}+\delta}{\delta}\right) \frac{M \mathbb{E}_{\tilde{\lambda}}\left(\left(L_{G}+\delta\right) T^{\tilde{\lambda}}\right)}{\Gamma(\widetilde{\lambda}+1)}, \quad \varepsilon \varrho(\omega),
$$

where

$$
M=\sup _{s \in[v, v+T]}\left(\frac{(s-v)^{\tilde{\lambda}}}{\mathbb{E}_{\tilde{\lambda}}\left(\left(L_{G}+\delta\right)(s-\nu)^{\tilde{\lambda}}\right)}\right),
$$

and $\delta$ is any positive constant.

Proof. Define the metric $d$ on $X$ in this way:

$$
d\left(x_{1}, x_{2}\right)=\inf \left\{D \in[0, \infty]: \frac{\left|x_{1}(\omega)-x_{2}(\omega)\right|}{\mathbb{E}_{\tilde{\lambda}}\left(\left(L_{G}+\delta\right)(\omega-v)^{\tilde{\lambda}}\right)} \leq D \varrho(\omega), \forall \omega \in I\right\} .
$$

Now, define the operator $\mathscr{A}: X \longrightarrow X$ such that

$$
(\mathscr{A} y)(\omega):=x(v)+\frac{1}{\Gamma(\widetilde{\lambda})} \int_{\nu}^{\omega}(\omega-s)^{\tilde{\lambda}-1} G(s, y(s)) \mathrm{d} s .
$$

It is easy to see that $d\left(\mathscr{A} y_{0}, y_{0}\right)<\infty$, and $\left\{y \in X: d\left(y_{0}, y\right)<\infty\right\}=X, \forall y_{0} \in X$.

Now, we prove that the operator $\mathscr{A}$ is a strictly contractive operator:

$$
\begin{aligned}
\left|\left(\mathscr{A} y_{1}\right)(\omega)-\left(\mathscr{A} y_{2}\right)(\omega)\right| & \leq\left|\int_{\nu}^{\omega} \frac{(\omega-\varsigma)^{\tilde{\lambda}-1}}{\Gamma(\tilde{\lambda})}\left\{G\left(\varsigma, y_{1}(\varsigma)\right)-G\left(\varsigma, y_{2}(\varsigma)\right)\right\} \mathrm{d} \varsigma\right| \\
& \leq \frac{1}{\Gamma(\widetilde{\lambda})} \int_{\nu}^{\omega}(\omega-\varsigma)^{\tilde{\lambda}-1}\left|G\left(\varsigma, y_{1}(\varsigma)\right)-G\left(\varsigma, y_{2}(\varsigma)\right)\right| \mathrm{d} \varsigma \\
& \leq L_{G} \int_{\nu}^{\omega}(\omega-\varsigma)^{\tilde{\lambda}-1} \frac{\left|y_{1}(\varsigma)-y_{2}(\varsigma)\right|}{\Gamma(\tilde{\lambda})} \mathrm{d} \varsigma \\
& \leq \frac{L_{G}}{\Gamma(\tilde{\lambda})} \int_{\nu}^{\omega}(\omega-\varsigma)^{\tilde{\lambda}-1} \frac{\left|y_{1}(\varsigma)-y_{2}(\varsigma)\right|}{\mathbb{E}_{\tilde{\lambda}}\left(\left(L_{G}+\delta\right)(\varsigma-v)^{\tilde{\lambda}}\right)} \mathbb{E}_{\tilde{\lambda}}\left(\left(L_{G}+\delta\right)(\varsigma-v)^{\tilde{\lambda}}\right) \mathrm{d} \varsigma \\
& \leq \frac{L_{G} d\left(y_{1}, y_{2}\right)}{\Gamma(\tilde{\lambda})} \int_{\nu}^{\omega}(\omega-\varsigma)^{\tilde{\lambda}-1} \varrho(\varsigma) \mathbb{E}_{\tilde{\lambda}}\left(\left(L_{G}+\delta\right)(\varsigma-\nu)^{\tilde{\lambda}}\right) \mathrm{d} \varsigma, \quad \text { for all } \omega \in I .
\end{aligned}
$$


Since $\varrho$ is nondecreasing, therefore,

$$
\begin{aligned}
\left|\left(\mathscr{A} y_{1}\right)(\omega)-\left(\mathscr{A} y_{2}\right)(\omega)\right| & \leq \frac{L_{G} d\left(y_{1}, y_{2}\right)}{\Gamma(\widetilde{\lambda})} \varrho(\omega) \int_{\nu}^{\omega}(\omega-\varsigma)^{\tilde{\lambda}-1} \mathbb{E}_{\tilde{\lambda}}\left(\left(L_{G}+\delta\right)(\varsigma-\nu)^{\tilde{\lambda}}\right) \mathrm{d} \varsigma \\
& \leq \frac{L_{G} d\left(y_{1}, y_{2}\right)}{L_{G}+\delta}\left(\mathbb{E}_{\tilde{\lambda}}\left(\left(L_{G}+\delta\right)(\omega-v)^{\tilde{\lambda}}\right)-1\right) \varrho(\omega) \\
& \leq \frac{L_{G} d\left(y_{1}, y_{2}\right)}{L_{G}+\delta}\left(\mathbb{E}_{\widetilde{\lambda}}\left(\left(L_{G}+\delta\right)(\omega-\nu)^{\tilde{\lambda}}\right)\right) \varrho(\omega), \quad \text { for all } \omega \in I,
\end{aligned}
$$

so that

$$
d\left(\mathscr{A} y_{1}, \mathscr{A} y_{2}\right) \leq \frac{L_{G}}{L_{G}+\delta} d\left(y_{1}, y_{2}\right)
$$

which means that the operator $\mathscr{A}$ is a strictly contractive operator. Now, since we have

$$
\left|{ }^{C} D_{\gamma, \omega}^{\tilde{\lambda}} x(\omega)-G(\omega, x(\omega))\right| \leq \varepsilon \varrho(\omega)
$$

then,

$$
|x(\omega)-\mathscr{A} x(\omega)| \leq \frac{\varepsilon}{\Gamma(\widetilde{\lambda})} \int_{\nu}^{\omega}(\omega-\varsigma)^{\tilde{\lambda}-1} \varrho(\varsigma) \mathrm{d} \varsigma,
$$

which implies that

$$
\begin{aligned}
\frac{|x(\omega)-\mathscr{A} x(\omega)|}{\mathbb{E}_{\tilde{\lambda}}\left(\left(L_{G}+\delta\right)(\omega-v)^{\tilde{\lambda}}\right)} & \leq \frac{\varepsilon}{\Gamma(\widetilde{\lambda}+1)} \varrho(\omega) \frac{(\omega-\nu)^{\tilde{\lambda}}}{\mathbb{E}_{\tilde{\lambda}}\left(\left(L_{G}+\delta\right)(\omega-\nu)^{\tilde{\lambda}}\right)} \\
& \leq \frac{\varepsilon M}{\Gamma(\widetilde{\lambda}+1)} \varrho(\omega) .
\end{aligned}
$$

Therefore,

$$
d(x, \mathscr{A} x) \leq \varepsilon \frac{M}{\Gamma(\widetilde{\lambda}+1)} .
$$

By employing Theorem 2, there is a solution $x^{*}$ of (16) such that

$$
d\left(x, x^{*}\right) \leq \varepsilon\left(\frac{L_{G}+\delta}{\delta}\right) \frac{M}{\Gamma(\widetilde{\lambda}+1)},
$$

so that

$$
\left|x(\omega)-x^{*}(\omega)\right| \leq\left(\frac{L_{G}+\delta}{\delta}\right) \frac{M \mathbb{E}_{\tilde{\lambda}}\left(\left(L_{G}+\delta\right) T^{\tilde{\lambda}}\right)}{\Gamma(\tilde{\lambda}+1)}, \quad \varepsilon \varrho(\omega),
$$

for all $\omega \in I$.

The following theorem represents the stability of (16) in the sense of UH.

Theorem 4. Assume $G: I \times \mathbb{R} \longrightarrow \mathbb{R}$ is continuous and satisfies

$$
\left|G\left(\omega, \kappa_{1}\right)-G\left(\omega, \kappa_{2}\right)\right| \leq L_{G}\left|\kappa_{1}-\kappa_{2}\right|, \quad \forall \omega \in I, \kappa_{i} \in \mathbb{R}, i=1,2 .
$$

If an absolutely continuous function $x: I \longrightarrow \mathbb{R}$ satisfies

$$
\left|{ }^{C} D_{\nu, \omega}^{\tilde{\lambda}} x(\omega)-G(\omega, x(\omega))\right| \leq \varepsilon,
$$

for all $\omega \in I$ and some $\varepsilon>0$, then there is a solution $x^{*}$ of (16) such that

$$
\left|x(\omega)-x^{*}(\omega)\right| \leq \varepsilon\left(\frac{L_{G}+\delta}{\delta}\right) \frac{M \mathbb{E}_{\tilde{\lambda}}\left(\left(L_{G}+\delta\right) T^{\tilde{\lambda}}\right)}{\Gamma(\tilde{\lambda}+1)},
$$

where

$$
M=\sup _{s \in[v, v+T]}\left(\frac{(s-v)^{\tilde{\lambda}}}{\mathbb{E}_{\tilde{\lambda}}\left(\left(L_{G}+\delta\right)(s-v)^{\tilde{\lambda}}\right)}\right),
$$

and $\delta$ is any positive constant.

Proof. The proof is similar to Theorem 3.

Remark 3. It should be noted that, in our analysis, we do not assume any condition on the constant $L_{G}$, unlike the case of Theorem 4.1 in [20], where the condition $0<\left(L_{G} r^{\lambda} /(\Gamma(\widetilde{\lambda}+\right.$ $1)))<1$ was a basic condition. 
Remark 4. Note that our results of the UH stability are some generalizations of the results obtained in [19].

The following theorem represents the stability of (16) in the sense of generalized UHR.

Theorem 5. Assume $G: I \times \mathbb{R} \longrightarrow \mathbb{R}$ is continuous and satisfies

$$
\left|G\left(\omega, \kappa_{1}\right)-G\left(\omega, \kappa_{2}\right)\right| \leq L_{G}\left|\kappa_{1}-\kappa_{2}\right|,
$$

for all $\omega \in I, \kappa_{i} \in \mathbb{R}, i=1,2$, and for some $L_{G}>0$. If an $a b$ solutely continuous function $x: I \longrightarrow \mathbb{R}$ satisfies

$$
\left|{ }^{C} D_{\nu, \omega}^{\tilde{\lambda}} x(\omega)-G(\omega, x(\omega))\right| \leq \varrho(\omega),
$$

for all $\omega \in I$, where $\mathrm{\varrho}(\omega)$ is a positive, nondecreasing, and continuous function, then there is a solution $x^{*}$ of (16) such that

$$
\left|x(\omega)-x^{*}(\omega)\right| \leq\left(\frac{L_{G}+\delta}{\delta}\right) \frac{M \mathbb{E}_{\tilde{\lambda}}\left(\left(L_{G}+\delta\right) T^{\tilde{\lambda}}\right)}{\Gamma(\tilde{\lambda}+1)} \varrho(\omega),
$$

where

$$
M=\sup _{s \in[\nu, \nu+T]}\left(\frac{(s-\nu)^{\tilde{\lambda}}}{\mathbb{E}_{\tilde{\lambda}}\left(\left(L_{G}+\delta\right)(s-v)^{\tilde{\lambda}}\right)}\right),
$$

and $\delta$ is any positive constant.

Proof. The proof is similar to Theorem 3.

Remark 5. Notice that, in our study of the generalized UHR stability, we do not assume any condition on $L_{G}$, unlike the case in Theorem 3.1 in [20].

Remark 6. Note that, in [19], the authors obtained stability results for differential equations with integer-order derivatives, while in our case, it is for fractional-order derivatives. In this sense, we generalized the interesting results in [19].

\section{Examples}

Two illustrative examples are given to show the validity of results.

Example 1. Consider equation (16) for $\tilde{\lambda}=0.6, v=0, T=9$, and $G(\omega, \kappa)=\omega^{2} \sin (\kappa)$.

We have

$\left|\omega^{2} \sin \left(\kappa_{1}\right)-\omega^{2} \sin \left(\kappa_{2}\right)\right| \leq 81\left|\kappa_{1}-\kappa_{2}\right|, \quad \forall \omega \in[0,9], \kappa_{1}, \kappa_{2} \in \mathbb{R}$.

Then, $L_{G}=81$.

Suppose that $x$ satisfies

$$
\left|{ }^{C} D_{0, \omega}^{0.6} x(\omega)-\omega^{2} \sin (x(\omega))\right| \leq 0.01(\omega+2),
$$

for all $\omega \in[0,9]$.
Here, $\varepsilon=0.01$ and $\psi(\omega)=\omega+2$. Using Theorem 3, there is a solution $x^{*}$ of the fractional differential equation and $M>0$ such that

$$
\left|x(\omega)-x^{*}(\omega)\right| \leq 0.01 M(\omega+2), \quad \forall \omega \in[0,9] .
$$

Example 2. Consider equation (16) for $\tilde{\lambda}=0.4, v=0, T=2$, and $G(\omega, \kappa)=\omega^{4} \cos (\kappa)$.

We have

$\left|\omega^{4} \cos \left(\kappa_{1}\right)-\omega^{4} \cos \left(\kappa_{2}\right)\right| \leq 16\left|\kappa_{1}-\kappa_{2}\right|, \quad \forall \omega \in[0,2], \kappa_{1}, \kappa_{2} \in \mathbb{R}$.

Then, $L_{G}=16$.

Suppose that $x$ satisfies

$$
\left|{ }^{C} D_{0, \omega}^{0.4} x(\omega)-\omega^{4} \cos (x(\omega))\right| \leq 0.01,
$$

for all $\omega \in[0,2]$.

Here, $\varepsilon=0.01$. Using Theorem 4 , there is a solution $x^{*}$ of the fractional differential equation and $M>0$ such that

$$
\left|x(\omega)-x^{*}(\omega)\right| \leq 0.01 \mathrm{M}, \quad \forall \omega \in[0,2] .
$$

\section{Conclusion}

It is known that, for the majority of fractional differential problems, a widely applicable general approach to determine the analytical solutions is not available. In this paper, we used a version of Banach fixed point theorem to prove that, under certain conditions, functions that satisfy some Caputo fractional differential equations approximately are close in some sense to the exact solutions of such kind of equations. In other words, we presented stability results for some Caputo fractional differential equations in the sense of UH, UHR, and generalized UHR. In our analysis, we used a new metric as a function of the Mittag-Leffler function. We end up with two examples that show the validity of our results.

\section{Data Availability}

No data were used to support this study.

\section{Disclosure}

An earlier version of this work has been presented as preprint in Authorea.

\section{Conflicts of Interest}

The authors declare that they have no conflicts of interest.

\section{Acknowledgments}

The authors extend their appreciation to the Deanship of Scientific Research at Jouf University for funding this work through research Grant no. DSR-2021-03-0121. 


\section{References}

[1] I. Podlubny, "Fractional differential equations," Mathematics in Science and Engineering, Vol. 198, Academic Press, San Diego, CA, USA, 1999.

[2] A. A. Kilbas, H. M. Srivastava, and J. J. Trujillo, Theory and Applications of Fractional Differential Equations, Vol. 204, Elsevier, Amsterdam, Netherlands, 2006.

[3] I. Koca, "Modelling the spread of Ebola virus with atanganabaleanu fractional operators," European Physical Journal Plus, vol. 133, no. 3, pp. 1-11, 2018.

[4] D. Baleanu, H. Mohammadi, and S. Rezapour, "Analysis of the model of HIV-1 infection of $\mathrm{CD}^{+}$T-cell with a new approach of fractional derivative," Advances in Difference Equations, vol. 2020, no. 1, 17 pages, Article ID 71, 2020.

[5] E. Uçar and N. Özdemir, "A fractional model of cancerimmune system with caputo and caputo-fabrizio derivatives," European Physical Journal Plus, vol. 136, no. 1, pp. 43-17, 2021.

[6] N. Brillouët-Belluot, J. Brzdek, and K. Ciepliński, "On some recent developments in ulam's type stability," Abstract and Applied Analysis, vol. 2012, Article ID 716936, 41 pages, 2012.

[7] S. András and A. R. Mészáros, "Ulam-Hyers stability of dynamic equations on time scales via picard operators," Applied Mathematics and Computation, vol. 219, no. 9, pp. 4853-4864, 2013.

[8] D. S. Cimpean and D. Popa, "Hyers-Ulam stability of Euler's equation," Applied Mathematics Letters, vol. 24, no. 9, pp. 1539-1543, 2011.

[9] B. Hegyi and S.-M. Jung, "On the stability of Laplace's equation," Applied Mathematics Letters, vol. 26, no. 5, pp. 549-552, 2013.

[10] S.-M. Jung, "Hyers-Ulam stability of linear differential equations of first order," Applied Mathematics Letters, vol. 17, no. 10 , pp. $1135-1140,2004$.

[11] T. M. Rassias, "On the stability of the linear mapping in Banach spaces," Proceedings of the American Mathematical Society, vol. 72, no. 2, pp. 297-300, 1978.

[12] A. Ahmadova and N. I. Mahmudov, "Ulam-Hyers stability of caputo type fractional stochastic neutral differential equations," Statistics \& Probability Letters, vol. 168, p. 108949, 2021.

[13] A. Ben Makhlouf, "Stability with respect to part of the variables of nonlinear caputo fractional differential equations," Mathematical Communications, vol. 23, no. 1, pp. 119-126, 2018.

[14] N. Eghbali, V. Kalvandi, and J. M. Rassias, "A fixed point approach to the Mittag-Leffler-Hyers-Ulam stability of a fractional integral equation," Open Mathematics, vol. 14, no. 1, pp. 237-246, 2016.

[15] J. Wang and Y. Zhou, "Mittag-Leffler-Ulam stabilities of fractional evolution equations," Applied Mathematics Letters, vol. 25, no. 4, pp. 723-728, 2012.

[16] J. Wang, L. Lv, and Y. Zhou, "Ulam stability and data dependence for fractional differential equations with caputo derivative," Electronic Journal of Qualitative Theory of Differential Equations, vol. 63, pp. 1-10, 2011.

[17] J. B. Diaz and B. Margolis, "A fixed point theorem of the alternative, for contractions on a generalized complete metric space," Bulletin of the American Mathematical Society, vol. 74, no. 2, pp. 305-310, 1968.

[18] T. S. Doan and P. E. Kloeden, "Semi-dynamical systems generated by autonomous caputo fractional differential equations," Vietnam Journal of Mathematics, pp. 1-11, 2019.
[19] Y. Başci, A. Misir, and S. Öğrekçi, "On the stability problem of differential equations in the sense of Ulam," Results Math, vol. 75, no. 1, pp. 1-13, 2020.

[20] J. Wang, L. Lv, and Y. Zhou, "New concepts and results in stability of fractional differential equations," Communications in Nonlinear Science and Numerical Simulation, vol. 17, no. 6, pp. 2530-2538, 2012.

[21] E. El-hady and S. Ögrekçi, "On Hyers-Ulam-Rassias stability of fractional differential equations with caputo derivative," Journal of Mathematics and Computer Science, vol. 22, pp. 325-332, 2021. 